\title{
Dynamic Nanostructuring of the Steel Surface Under High-Speed Thermal Heating
}

\author{
Andrey Brover $^{1 *}$, Galina Brover $^{1}$, Olga Moysova ${ }^{1}$, and Valeriya Yankovskaya ${ }^{2}$ \\ ${ }^{1}$ Don State Technical University, Physical and Applied Materials Science Department, Gagarin Sq.,1, \\ Rostov-on-Don, Russia \\ ${ }^{2} \mathrm{Kiev}$ College of Mechanics and Technology, Metallurgy and Materials Science Department, Kharkiv \\ Hwy.,15, Kiev, Ukraine
}

\begin{abstract}
The research depicts structural picture of the steel surface layers after pulsed laser treatment with surface reflow. There are factors contributing to the dispersion of forming crystals to the nanoscale level. There is also found interdependence of nanostructure and laser-irradiated products strength characteristics.
\end{abstract}

\section{Introduction}

In modern engineering industry much attention is paid to the quality of surface layers. While hardened products are used they are exposed to significant fluctuations in temperature and stress, which cause premature failure of products due to destruction. Therefore, one of the most promising and rapidly developing methods of heat treatment of metal surface is the impulsive laser technology, which significantly improves the performance of steels and alloys surface layers.

Laser surface treatment of engineering materials is generally local. The size of the zones of pulsed laser action is a few millimeters (spot diameter $\sim 1-5 \mathrm{~mm}$ ), the depth of the zone of thermal influence $(\sim 50-150 \mu \mathrm{m})$ [1-3].

\section{Research methods}

The samples were irradiated on materials of various chemical compositions (further marking in accordance with the German standard (DIN), highlighted in bold). Pulsed laser surface processing was carried out at laser equipment "Kvant-16" with a change in the radiation power density in the range of $80-150 \mathrm{MW} / \mathrm{m}^{2}$. The degree of beam defocus (3$6 \mathrm{~mm})$ and the duration of radiation $(1-6) \cdot 10^{-3} \mathrm{~s}$ allowed us to vary the density of radiation power within wide limits.

Surface processing was applied on more than 7 samples of $10 \times 10 \times 15 \mathrm{~mm}$ for each hardening. Measuring different values, their average measures, standard deviations and

\footnotetext{
* Corresponding author: brover@mail.ru
} 
trust intervals were determined at the level of reliability $\mathrm{P}=0,95$ (Student's criteria was 2,447).

Metallographic studies were performed on cross-sections. The microstructure photo was made with a "Neophot-21" microscope. The thin structure of the processing areas was analyzed using a "Hitachi TM-1000" scanning electron microscope.

Identification of phase compounds in the areas of thermic influence was made by diffraction x-ray depth analysis, after electrolytic polishing of samples and metallographic control of the studied surface structure. The study was carried out on defractometer "DRON-0,5" in filtered $\mathrm{FeK}_{\alpha}$ - radiation with registration of the intensity of scintillators. X-ray lines profiles scanning was a nonstop recording at a speed of $1 \mathrm{deg} / \mathrm{min}$. Nanonstructural research was carried out with a complex scanning probe microscope "NanoEducator".

\section{Discussion of results}

Metallographic research of laser-irradiated steels shows a pronounced heterogeneity of the structure, composition and properties of the irradiated spot because of hyper-high heating and cooling, as well as significant temperature and stress gradients (see below). Thus, a structure is formed on the surface of materials, the features of which are determined by the degree of completion of phase and structural transformations during heating and cooling.

Generally, the irradiated layer has three distinct completely different phase transformations zone: melting zone; zone of quenching from a solid state; transitional or heat-affected zone, formed by heating the basic metal due to heat remove from the irradiated spot. Laser treatment of metals with surface melting opens up new opportunities for obtaining a qualitatively different structural state in alloys compared to traditional processes of thermal hardening with concentrated energy flows [4-7].

At the same time, the following effects contribute to an increase in the level of hardening of laser-irradiated steels:

- martensitic transformation during cooling after laser quenching;

- increased density of the resulting defects in the crystal structure, 10 times exceeding the same for volume-hardened metal;

- chemical and structural significant inhomogeneity of solid solutions, resulting from slowing down the diffusion processes of redistribution of alloying elements during highspeed heating processes.

It should be noted that laser processing with melting of the surface forms the final structure at the cooling stage of the molten metal. After the light beam starts on the material, a thin layer of liquid phase $(10-30 \mu \mathrm{m})$ appears in the central part of the irradiated spot, which is characterized by dendritic forms of crystal growth. Their shape largely determines the surface quality indicators after laser treatment, so they are one of the objects of research when structuring various materials under laser. Dendrites grow in the direction of a negative temperature gradient, that is from the surface of the product to the depth. At the same time, the high crystallization rate contributes to a significant dispersion of crystals in the cross-section up to the nanoscale level.

This can be confirmed by microstructural research of the surface layers of steel $\mathbf{1 . 3 3 4 3}$ after impulsive laser treatment with surface melting. The micro picture (figure 1), shows the mosaic structure of the structure in the fused part of the spot. At the same time, revealed by thermal etching, such features of the fused zone structure as the serration of grain boundaries and the presence of slip bands should be noted. 


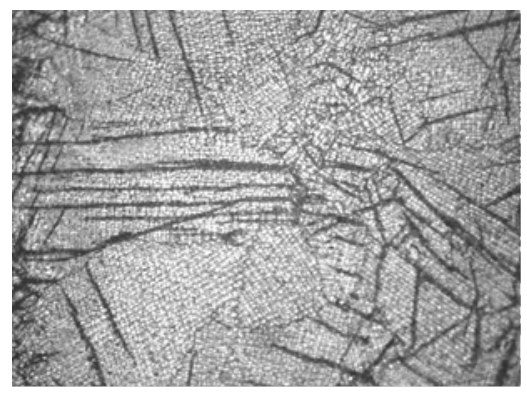

Fig. 1. Microstructure (top view) of the fused zone on steel $\mathbf{1 . 3 3 4 3}(\times 900)$

This effect may be related to a process similar to dynamic high-temperature deformation. The curve of grain boundaries in the form of serration is probably a result of border migration due to significant micro-stresses and plastic deformation in microvolumes that occur during laser processing.

During laser melting of the metal surface, that is when a layer of solidified melt appears in the irradiation spot, the main source of local plastic deformation is thermal stresses during crystallization, as well as stresses arising at the border of the irradiated zones of the source metal [8-10]. Hydrodynamic mixing of the melt under the thermic capillary forces resulting from inhomogeneous surface heating makes an additional contribution to the appearance of stresses and the formation of the observed mosaic structure consisting of subgrains (blocks).

Thus, in the course of research, it was found that in the zones of laser quenching from the liquid state due to temperature and concentration gradients, there occur a high degree of non-equilibrium of the liquid phase, bifurcation instability of the melt and the transition of the laminar flow of the liquid to a turbulent one. The resulting circulation vortices, which break up into a number of sub vortices, provide energy dissipation at various levels, abnormal mass transfer flows, and dispersion of growing crystals, including nanostructural effects.

The control parameter in this case is the vibrational pressure gradient at the solid-liquid interface, which, when the critical level is reached, becomes a parameter that controls convective and anomalous diffusion flows, providing dispersion of growing crystals. The convective flow is responsible for the dispersion in the bath of the melt rich in dissolved elements. The small size of the crystallites of the molten metal leads to the fact that a significant portion of the material (up to 50\%) is occupied by inter crystalline boundaries. This radically changes the mechanisms responsible for the formation of macroscopic characteristics of the laser-fused metal. The observed features of structure formation at high cooling rates can be clarified by analyzing the crystallization kinetics.

To make a reliable calculation of the velocity of liquid-solid boundary movement $\left(V^{-n}\right)$ and identify the following influence on structure formation, we will compare it with the type and typical size of the structure under laser processing.

Let's consider a case of the fused zone of the hardened spot structure, when in the cross section columnar crystals look like equally axed grains consisting of separate blocks in a certain way within each grain, that is, the central part of the spot has a mosaic structure. Using the correlation between the cooling rate $(V)$ and the distance between the axes of dendrites of the $\mathrm{i}$ order (d) for a number of alloys in the specified range of cooling rates, we determine the values of the local crystallization rate $\left(V^{-n}\right)$ from the experimentally established dependence [4]:

$$
d=A \cdot V^{-n}
$$

$A, n$ are the constants of the material. 
The values of constants $A$ and $n$ in (1) for some materials are shown in table 1 .

Table 1. The values of the constants for different materials [13]

\begin{tabular}{|c|c|c|c|c|c|c|}
\hline Material & $\mathbf{1 . 3 3 5 5}$ & $\mathbf{1 . 2 3 7 9}$ & $\mathbf{1 . 4 8 7 8}$ & Nickel & $\mathbf{1 . 3 5 0 5}$ & Iron \\
\hline$A, \times 10^{-2}$ & 1,6 & 1,7 & 2,2 & 2,5 & 3,6 & 3,8 \\
\hline$n$ & 0,45 & 0,45 & 0,45 & 0,45 & 0,45 & 0,45 \\
\hline
\end{tabular}

Micropicturing of laser reflow sites with a mosaic dendritic structure on a scanning electron microscope (figure 2, a), as well as profile of the height distribution of surface irregularities, obtained using the graphical program for analyzing two-dimensional image height fields Gwyddion (figure 2, b), allow us to measure $d$.

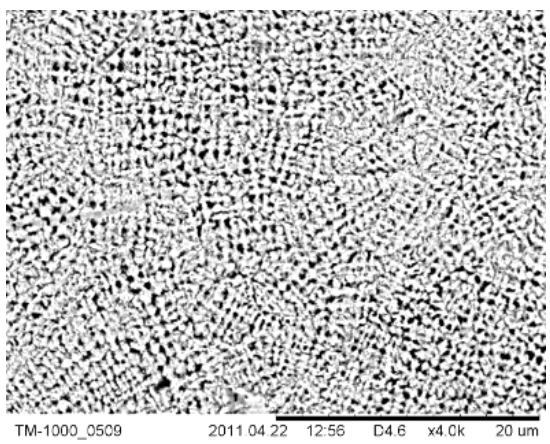

a

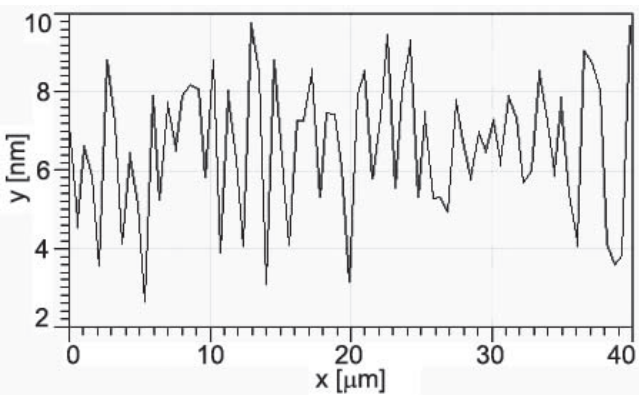

b

Fig. 2. Microstructure of the fused zone on steel 1.2379 (a), profile of heights of dendrites (b)

Using (1), we get the cooling rate after laser exposure in the central zone of the spot for various materials about $10^{5}-10^{6} \mathrm{~K} / \mathrm{s}$.

At such cooling rates, columnar crystals should grow, completely devoid of lateral branches or surface dendrites in the form of spherulites [11, 12]. The results of metalgraphic studies confirm this conclusion.

With a further increase in the cooling rate above $10^{6} \mathrm{~K} / \mathrm{s}$, a complete degeneration of dendritic growth and stabilization of the flat crystallization occurs.

This is confirmed by the results of X-ray structural analysis of steels after laser treatment with surface melting. Figure 3 shows fragments of X-ray of irradiated steel $\mathbf{1 . 0 5 0 3}$ in the initial annealed state. Laser treatment was performed both with reflow (figure 3, curve 2) and without surface reflow (figure 3, curve 1). It can be seen that during laser quenching from a liquid state, an abnormal ratio of the intensities of the austenite difraction lines is observed, which indicates the appearance of texture in the surface layers of irradiated steels.

The described effect has a positive effect on the properties of products after laser processing, in particular, it reduces the friction coefficients in the friction pairs, if the texture type agrees with the type of stress state of the hardened products under operation.

It was found that the micro hardness in the zone of laser quenching from the liquid state is sufficiently high on both carbon and alloy steels and is 3-6 HPa. It can be expected that the formation of a mosaic structure in irradiated zones with a large extent of the total surface of sub-boundaries, most of which are "semipermeable" barriers, will contribute to stress relaxation and reduce the risk of cracking [18].

Thus, it can be concluded that rapid crystallization from the melt during processing with concentrated energy flows opens up new opportunities for obtaining a qualitatively different structural state in alloys compared to traditional metallurgical processes. 


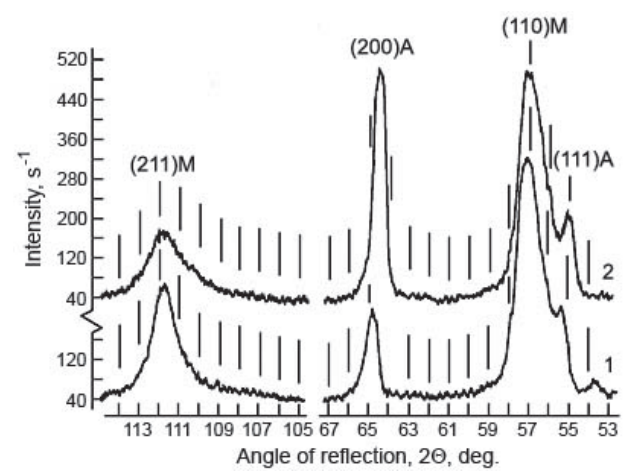

Fig. 3. Roentgenogram fragments of annealed steel $\mathbf{1 . 0 5 0 3}$ after laser treatment without reflow (1) and with surface reflow (2)

The dispersion described above in the surface layers of laser irradiated steel $\mathbf{1 . 3 3 5 5}$ was studied by using scanning probe microscopy. The results of experiments (figure 4) revealed nanostructural effects. As can be seen from the figure, pulsed laser processing leads to the formation of a structural pattern with the size of elements $100 \mathrm{~nm}$ (or less).

However, this order is typical:

- for the cross-section of crystals, that is in the direction perpendicular to the action of the laser beam, which is clearly shown in figure 4;

- for the axial direction of crystal growth (perpendicular to the irradiation surface). This is confirmed by the histogram of the distribution of heights of structural elements on the studied surface, the relief (located on the right of figure 4, a).

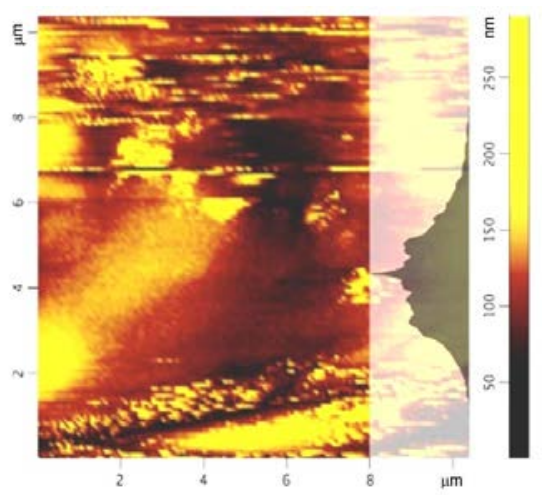

a

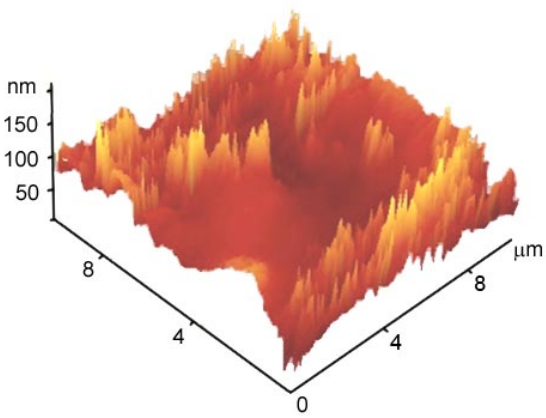

b

Fig. 4. Atomic force microscopy of laser-irradiated surface of steel 1.3355. Two-dimensional (a) and three-dimensional (b) image of the relief after scanning the area of $10,5 \times 10,5 \mu \mathrm{m}$.

Based on modern ideas about nanostructured materials [13-17], this effect is a direct confirmation of a peculiar and non-trivial "dualism" of the properties of laser strengthened layers, which high hardness attends a definite reserve of viscosity that is not worse than bulk-heat-strengthened steels.

Apparently, when the nanoscale structure described is formed in the irradiated layers, a significant stress gradient occurs (phase, mechanical, etc.), which contributes to the formation of a developed dislocation substructure that extends deep into the material. As a result, a structural matrix is created, with lots of "semipermeable" barriers for dislocation movement, which contributes to the relaxation of peak stresses not by the beginning and spreading of cracks, but by transfer of stresses to neighboring micro-volumes of crystals. 
In order to confirm these considerations, wear resistance tests were performed on samples made of steel $\mathbf{1 . 3 3 5 5}$ after pulse laser treatment with surface reflow and after standard heat treatment-quenching from $1220^{\circ} \mathrm{C}$ and three - fold tempering. The results showed a 2,5-times superiority of laser-irradiated samples after 10 minutes of testing and up to a 7-times advantage after a 120-minute experiment [18, 19].

The review of the surface of volume-hardened samples after friction tests shows that they have a damaged surface contact with the areas of setting and displacement of the metal. On the contrary, samples subjected to laser reflow had a fairly clean surface after testing.

However, it should be noted that the structural strength of laser-fused zones has a sufficiently high values only when they are used under compressive stress. If the use of irradiated products is associated with tensile stresses, this property is significantly reduced.

\section{Conclusion}

The research allows us to conclude that high-speed laser treatment of steel with surface melting due to high cooling speed leads to the generation of stress waves that accelerate the mass transfer processes and lead to high-speed local plastic deformation of the surface layer micro-volumes. Under these conditions, a nanostructural pattern is formed, which contributes to increasing the strength characteristics of laser-irradiated products.

\section{References}

1. A.G. Grigoryanc, I.N. Shiganov, A.I. Misyurov, Technological processes of laser processing (2006)

2. M. Katancik, S. Mirzababaei, M. Ghayoor, S. Pasebani, J. of Alloys and Compounds, 849, $156319(2020)$

3. J. Tu, KF. Zhou, ZM. Zhou, C. Huang, HL. Tang, Materials Characterization, 128, 63 (2017)

4. D.M. Gureev, Phys. and Chem. of Materials Processing, 6, 126 (1994)

5. A.V. Shelyakov, N.N. Sitnikov, I.A. Khabibullina, R.V. Sundeev, O.N. Sevryukov, Phys. Sol. St., 62(6), 937 (2020)

6. G.V. Lomaev, E.V. Haranzhevskij, Met. Sci. and Heat Treat. of Met., 3, 27 (2002)

7. X. Zhao, YH. Lv, SY. Dong, SX. Yan, XT. Liu, YX. Liu, P. He, TS. Lin, BS. Xu, HS. Han, Opt. and Laser Techn., 132, 106487 (2020)

8. A.V. Brover, G.I. Brover, Yu.A. Kornilov, Izvestiya VolGTU, 2(181), 120 (2016)

9. C. Zinn, M. Bobbert, C. Dammann, Z Wang, T. Troster, R. Mahnken, G. Meschut, M. Schaper, Composites part b-engineering, 151, 173 (2018)

10. Yu.A. Samojlovich, Crystallization of the ingot in the electromagnetic field (1986)

11. L.G. Korshunov, N.L. Chernenko, J. Surf. Investigat. 14(3), 632 (2020)

12. I.S. Miroshnichenko, Quenching from the liquid state (1982)

13. P. Ohodnicki, J. Egbu, Y. Yu, J. Baltrus, N. Aronhime, Y. Krimer, P. Anand, K. Byerly, M.E. McHenry, J. of Alloys and Compounds, 834, 155038 (2020)

14. L. Zhu, S.N. Liu, S. Lan, Y.M. Xu, Li, C. Li, H. Zheng, S.S. Jiang, J.R. Men, XL. Wang, F.M. Pan, J. of Alloys and Compounds, 823, 153911 (2020)

15. D.I. Ryzhonkov, Nanomaterials (2008)

16. A. Samanta, Qh. Wang, S.K. Shaw, Ht. Ding, J. Las. Appl., 31(2), 022515 (2019)

17. Qh. Wang, A. Samanta, SK. Shaw, H. Hu, H. Ding, Appl. Surf. Sci., 507, 145136 (2020)

18. A.V. Brover, G.I. Brover, O.V. Shevcova, Strength. technol. and Coat., 2(122), 21 (2015)

19. S. Graf, Adv. Opt. Technol., 9(1-2), 11 (2020) 